\title{
Recent advances in the combination of QCD and EW corrections to the Drell-Yan processes
}

\author{
Alessandro Vicini* \\ Dipartimento di Fisica, Università degli Studi di Milano and INFN, Sezione di Milano, Via \\ Celoria 16, 20133 Milano (Italy) \\ E-mail: alessandro.vicini@mi.infn.it
}

\section{Giovanni Balossini and Guido Montagna}

Dipartimento di Fisica Nucleare e Teorica, Università di Pavia, and INFN, Sezione di Pavia, via A. Bassi 6, 27100 Pavia, Italy

Email: giovanni.balossini@pv.infn.it

Email: guido.montagna@pv.infn.it

\section{Carlo Michel Carloni Calame}

School of Physics \& Astronomy, University of Southampton, Southampton SO17 1BJ, UK

Email: c.carloni-calame@phys.soton.ac.uk

\section{Mauro Moretti}

Dipartimento di Fisica, Università di Ferrara, and INFN, Sezione di Ferrara, via Saragat 1, 44100 Ferrara, Italy

Email: mauro.moretti@fe.infn.it

\section{Oreste Nicrosini and Fulvio Piccinini}

INFN, Sezione di Pavia, via A. Bassi 6, 27100 Pavia, Italy

Email: oreste.nicrosini@pv.infn.it

Email: fulvio.piccininiepv.infn.it

\section{Michele Treccani}

Departamento de Física Teórica y del Cosmos, CAPFE, Universidad de Granada, E-18071 Granada, Spain

Email: treccani@ugr.es

The issue of matching fixed order calculations with all order results and the recipes to combine EW and QCD corrections in the simulation of the Drell-Yan processes are reviewed.

RADCOR 2009 - 9th International Symposium on Radiative Corrections (Applications of Quantum Field Theory to Phenomenology),

October 25 - 302009

Ascona, Switzerland

\footnotetext{
* Speaker.
} 
The Drell-Yan (DY) processes allowed the discovery of the $W$ and $Z$ bosons in 1983 at the CERN SPS. They have provided at the Tevatron important tests of the Standard Model including measurements of the $W$ and $Z$ masses and width, of the weak mixing angle and constraints on the parametrization of the proton parton densities. Given the high precision of the theoretical prediction for their cross section, it has been proposed to use these processes at the LHC as standard candles, i.e. as benchmarks for the normalization of other physical cross sections and for the monitoring of the machine luminosity. A detailed review of the progress over the last three decades in the calculation of all the relevant QCD and EW perturbative corrections and in their implementation in computer codes can be found in [1].

Since the DY processes allow very high precision measurements, it is important to understand which level of accuracy can be attained in the theoretical calculations and whether for instance a determination of the cross sections at the $1 \%$ level, or the extraction of the $W$ mass with $15 \mathrm{MeV}$ of error, can be meaningfully obtained.

One issue which is already known for more than 15 years is the problem of matching fixed order QCD calculations with the description of multiple gluon emission from the initial state. Since there are different simulation tools available, it is highly desirable to have a tuned comparison that shows the numerical impact of the different approximations used in these programs. A second relevant topic is the inclusion of EW corrections in a purely QCD simulation. In these proceedings we will address some open questions related to the first point, which are currently under study in a workshop started in Milano last March [2], following preliminary studies during an INFN Workshop held in Frascati [3] and at the Les Houches Workshop "Physics at TeV Colliders" [4] . Secondly we will report the results of some detailed study concerning the combination of EW and QCD corrections.

\section{QCD matching}

The QCD corrections have an important role in the DY processes which can be demonstrated with three peculiar examples. 1) They yield a large K-factor, of the order of $20 \%$ of the total cross section within the usual acceptance cuts at Tevatron and at LHC, which was discovered in the first fixed order perturbative QCD calculations. 2) On the other hand the multiple gluon emission has a dramatic impact on the shape of any distribution at hadron colliders. Observables like the invariant/transverse mass or the lepton transverse momentum distributions are sensibly broadened by the gluon emission. 3) The gauge boson transverse momentum distribution is due to initial state radiation; its spectrum is divergent for low transverse momenta in fixed order calculations; the finiteness of the distribution is restored after the resummation to all orders of the relevant logarithmic terms.

The descritpion of multiple gluon emission can be obtained either in a Montecarlo simulation with a Parton Shower approach or computing at a given order the coefficient which appear in standard resummation formalism. In both cases we should specify: 1) a procedure for the matching of fixed order with all order results; 2) the accuracy that can be reached in that approach.

The inclusion of multiple initial state gluon emission has a link with the non-perturbative dynamics of QCD at low transverse momentum scales, whose modeling dependence should be quantified. 
From the point of view of the resummed results, there are two codes that implement the formalism for the resummation of soft-gluon emission: RESBOS $[5,6]$ and the code by the Florence group [7,8].

RESBOS resums à-la Collins-Soper-Sterman the leading (LL) and next-to-leading logarithms (NLL) of the gauge boson transverse momentum $\left(p_{\perp}^{V}\right)$. It includes the full NLO results and part of the available NNLO results. The matching procedure finds the point where fixed and resummed $\left(p_{\perp}^{V}\right)$ distributions cross each other, typically at a scale of 25-30 GeV. Below (above) the matching scale the resummed (fixed order) results are used. The fact that the first derivative of the matched distribution is not necessarily continuous at the matching point belongs to the ambiguity of the matching procedure. The non-perturbative effects are parametrized by three coefficients which appear in the resummation factor, whose values can be fitted from the data.

The code by the Florence group resums to all order the soft gluon emission working in the impact parameter space. The final result is factorized into the product of two terms: 1) the exponential factor which is universal, which includes all the resummed terms and is free of initial state collinear singularities; 2) the hard scattering function which describes the specific process. This splitting requires the appearance of an auxiliary scale, called resummation scale, which should not be confused with the factorization scale of collinear singularities. The resummation scales parametrizes the ambiguity inherent to the matching procedure and should be reduced by the introduction of higher order corrections. In its published version the code include the NLO+LL corrections. Based on the recent full NNLO results, it is in preparation an updated version that should reach the NNLO+NLL accuracy. Also in this code it is possible to introduce in the exponential factor non-perturbative coefficients, whose values can be fitted from the data.

The two codes described above integrate over the radiation in the soft approximation and are exclusive for all the leptonic variables. The Montecarlo approach instead describe in a fully exclusive way the emission of any additional parton. One advantage is that additional cuts on the hadronic variables can be imposed in a very simple way. Two recipes have been proposed to match the QCD-PS with exact NLO results: the one implemented in MC@NLO [9] and the one of POWHEG [10]. In both cases the problem is the merging of two results, avoiding the double counting of the common terms (the first leading log present in the NLO calculation and also simulated by the QCD-PS).

In MC@NLO the fixed order results are matched with the QCD-PS of HERWIG [11]. The double counting problem is solved by introducing HERWIG-dependent subtraction terms. These terms are evaluated at a matching scale that has to be specified.

In the POWHEG method the first emission is by construction the hardest one and has NLO accuracy. The value of the transverse momentum scale of the first emission is communicated to any vetoed Parton Shower (HERWIG, PYTHIA [12],...) that will emit additional partons at most up to that scale: the ordered emission, obtained imposing that the first emission is the hardest one, is by construction free of double counting. There is not a fixed matching scale, but one can rather say that on a event-by-event basis the virtuality of the first parton plays this role.

Differences between the POWHEG and the MC@NLO prescriptions are of higher order, i.e. of NLL accuracy. Additional subleading differences may appear in POWHEG using different Parton Shower, because of the different ordering of the multiple emissions.

QCD-PS programs encode, with some dedicated model, the non-perturbative effects on the 
low transverse momentum radiation. MC@NLO and POWHEG+HERWIG have the same content in this sector, whereas differences can be observed comparing POWHEG+HERWIG and POWHEG+PYTHIA.

In summary, we have at disposal at least four codes that share NLO+LL accuracy. Differences between them are at the level of higher order terms (at present there is a partial, almost complete, inclusion of NNLO terms in RESBOS and in progress is the full inclusion in the Florence code) or at the level of NLL terms (fully resummed in RESBOS, in progress in the Florence code, partially included with different recipes in MC@NLO and in POWHEG).

Tuned comparisons are technical checks that should guarantee the absence of bugs in code that share exactly the same formal accuracy (typically at fixed order), that use the same input parameters (couplings, masses, renormalization/factorization scales) and the same acceptance cuts: in fact under such conditions one would expect identical results, within the numerical accuracy. In the "W-mass workshop" a systematic series of comparisons has been started. After the benchmarks provided by these exercises will be available, one will possibly appreciate the numerical impact of higher-order/subleading terms of the four codes mentioned above. It will be very interesting to study the differences in the predictions of the lepton transverse momentum and the related transverse mass spectra, which are crucial for the extraction of the $W$ mass.

\section{Combination of $\mathrm{QCD}$ and $\mathrm{EW}$ corrections}

It is well known that EW corrections [13] have an important role in the precise determination of all the relevant leptonic observables of the DY processes (transverse lepton momentum, invariant and transverse mass), and in turn they have a sizeable impact on the determination of important constants like $W$ and $Z$ masses and decay widths. Since initial state multiple gluon emission is fundamental to obtain the lowest realistic description of the shape of several distributions, it will also significantly affect the fixed order EW corrections. The interplay between QCD and EW corrections starts at $\mathscr{O}\left(\alpha \alpha_{s}\right)$; since a full exact calculation is missing, it is important to find recipes to include, with some approximation, the bulk of these terms $[4,15,16,17,18]$.

In ref. [1] two formulae have been devised to combine EW and QCD corrections, including the fixed order results $\mathscr{O}(\alpha)+\mathscr{O}\left(\alpha_{s}\right)$ and the bulk of the mixed $\mathscr{O}\left(\alpha \alpha_{s}\right)$ terms

$$
\begin{aligned}
{\left[\frac{d \sigma}{d \mathscr{O}}\right]_{\mathrm{QCD} \& \mathrm{EW}}=} & \left\{\frac{d \sigma}{d \mathscr{O}}\right\}_{\mathrm{QCD}}+\left\{\left[\frac{d \sigma}{d \mathscr{O}}\right]_{\mathrm{EW}}-\left[\frac{d \sigma}{d \mathscr{O}}\right]_{\mathrm{Born}}\right\}_{\mathrm{HERWIG} \mathrm{PS}} \\
{\left[\frac{d \sigma}{d \mathscr{O}}\right]_{\mathrm{QCD} \otimes \mathrm{EW}}=} & \left(1+\frac{[d \sigma / d \mathscr{O}]_{\mathrm{MC} @ \mathrm{NLO}}-[d \sigma / d \mathscr{O}]_{\mathrm{HERWIG} \mathrm{PS}}}{[d \sigma / d \mathscr{O}]_{\mathrm{LO} / \mathrm{NLO}}}\right) \times \\
& \times\left\{\frac{d \sigma}{d \mathscr{O}_{\mathrm{EW}}}\right\}_{\mathrm{HERWIG} \mathrm{PS}},
\end{aligned}
$$

In eq. 2.1, which we call additive combination, the EW corrections are convoluted with the QCDPS by HERWIG: the exact $\mathscr{O}(\alpha)$ corrections are enhanced by the QCD leading-log corrections. In eq. 2.2, which we call factorized combination, the EW corrections are first convoluted with the QCD-PS by HERWIG and then are multiplied by a correction factor $\left(1+\delta_{Q C D}\right)$ : in this way the 


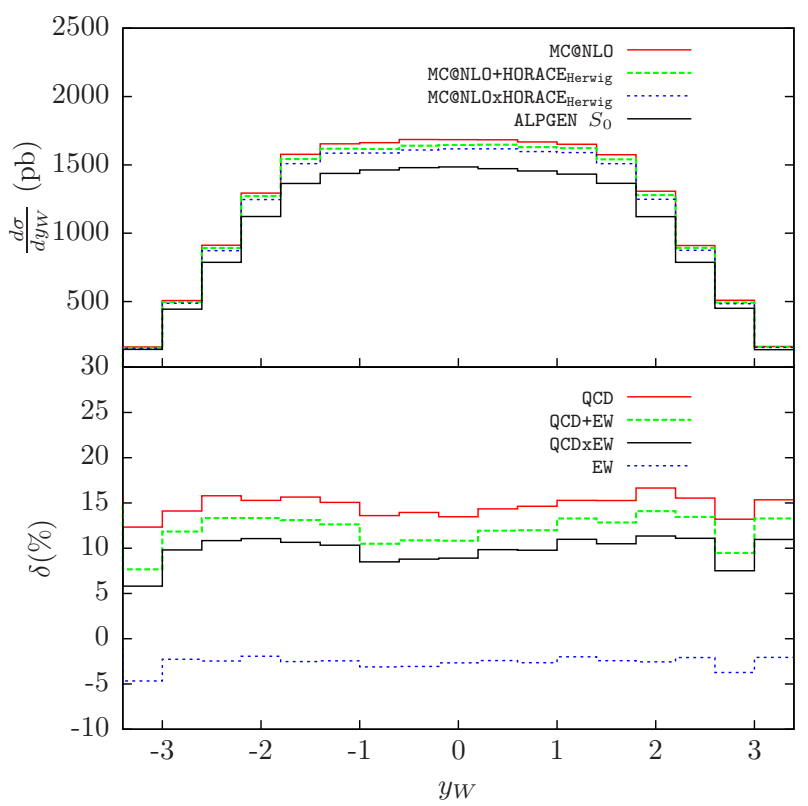

Figure 1: Different corrections to the $W$ rapidity distribution at the LHC.

NLO-QCD accuracy is reached; this factorized formula includes the bulk of the reducible factorizable terms of $\mathscr{O}\left(\alpha_{s}^{2}\right)$; the EW corrections are multiplied not only by the leading logs, but also by the constant part of the NLO-QCD calculation. In summary, in the two recipes higher order terms

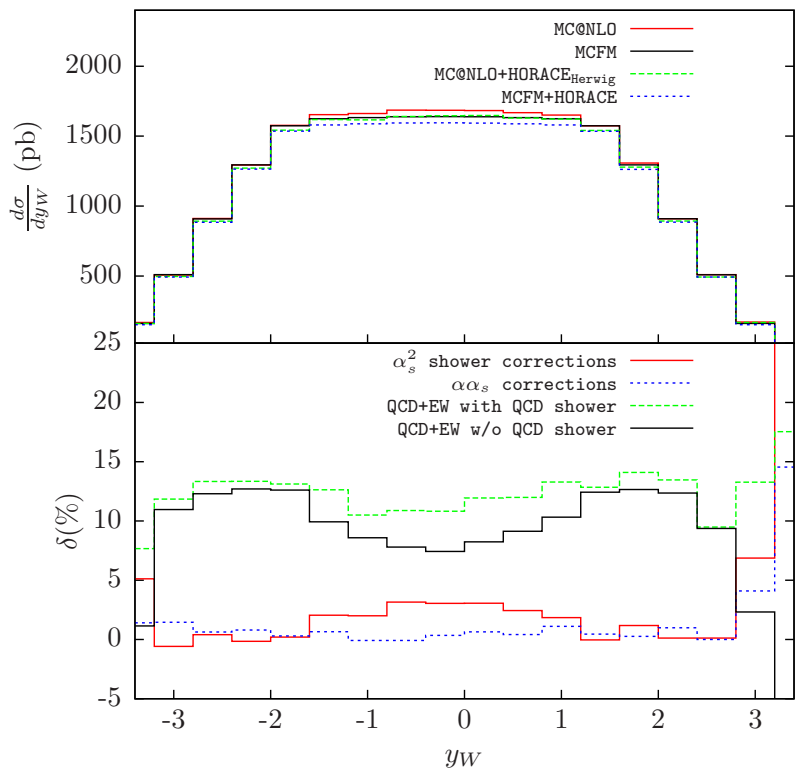

Figure 2: Different corrections to the $W$ rapidity distribution at the LHC.

of $\mathscr{O}\left(\alpha_{s}^{2}\right)$ and $\mathscr{O}\left(\alpha \alpha_{s}\right)$ are included in different ways.

In figures $(1,2)$ it is shown the effect, in various approximations, of different corrections to the $W$ rapidity distribution. In fig. 1, the results by MC@NLO are larger than the pure QCD Parton Shower (ALPGEN $S_{0}[19]$ ) by the NLO K-factor of about $15 \%$. More interesting the additive and 


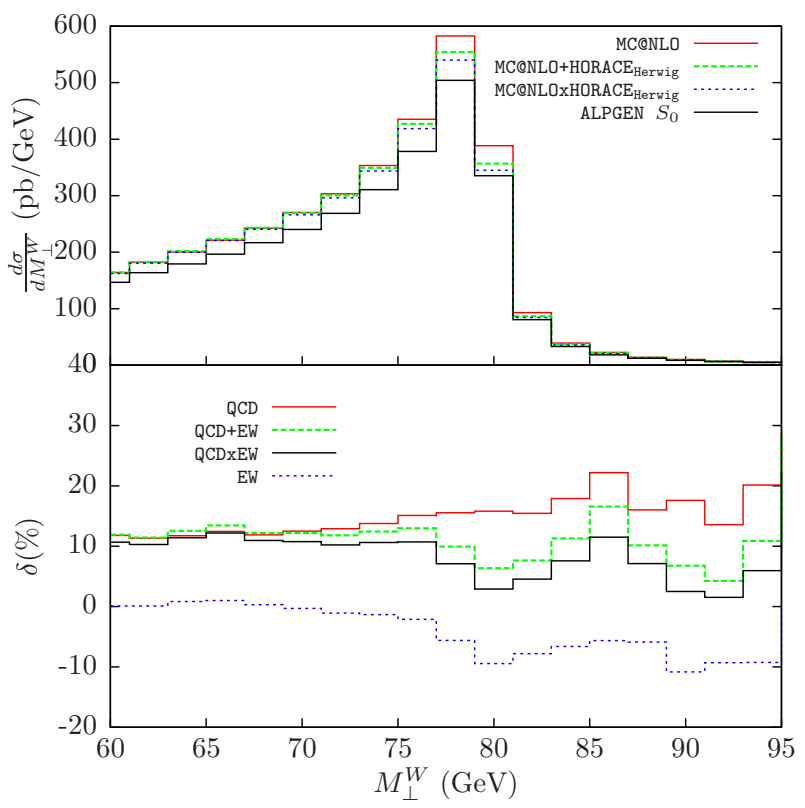

Figure 3: Different corrections to the $W$ transverse mass distribution at the LHC.

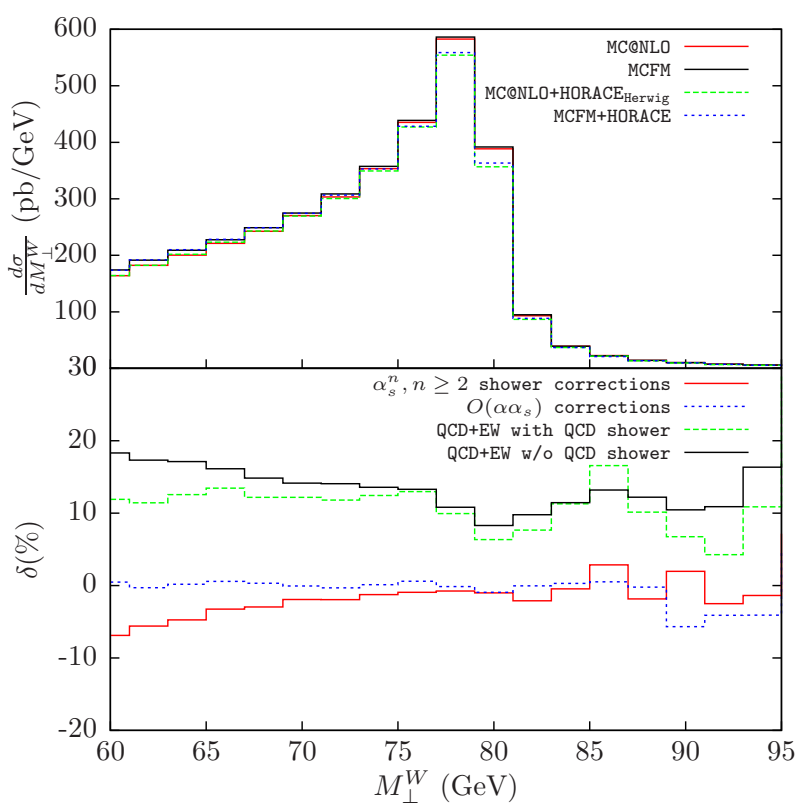

Figure 4: Different corrections to the $W$ transverse mass distribution at the LHC.

factorized recipes to combine EW+QCD effects differ at the level of 2-3\%. In fig.2 we disentangle individual contributions: the pure QCD Parton Shower higher orders are positive (MC@NLO is larger than MCFM [20] that only has fixed NLO-QCD); the EW corrections are negative and in presence of the Parton Shower their effect is reduced and broadened. In figures $(3,4)$ it is shown the effect, in various approximations, of different corrections to the $W$ transverse mass distribution. In fig. 3, the results by MC@NLO are larger than the pure QCD Parton Shower (ALPGEN $S_{0}$ ) with a NLO K-factor that ranges from 10 to $20 \%$. Again, the additive and factorized recipes to 
combine EW+QCD effects differ at the level of 2-3\%, in the peak region. In fig.4 we disentangle individual contributions: the pure Parton Shower higher order are negative (MC@NLO is smaller than MCFM). It is worth noticing that for the first bins close to the kinematical boundary $\left(M_{T}^{W}=\right.$ $50 \mathrm{GeV}$, not shown in the figure) the prediction by MCFM is negative. This is a well known effect already discussed in $[8,21]$, and due to perturbative instabilities of the NLO calculation. As a consequence, the results by MCFM around the jacobian peak appear to be slightly larger than the corresponding ones by MC@NLO, without contradicting the results for the integrated cross sections, obtained in [1]. Again EW corrections are negative. We also note that the purely mixed $\mathscr{O}\left(\alpha \alpha_{s}\right)$ in units Born+QCD-PS are small. To emphasize the differences between the additive and factorized recipes, we show in fig. 5 the ratio of the two predictions for the transverse mass distribution: we observe a different overall normalization, which is mostly related to the different QCD $\mathscr{O}\left(\alpha_{s}^{2}\right)$ terms; we see also a non-trivial shape difference in the peak region.

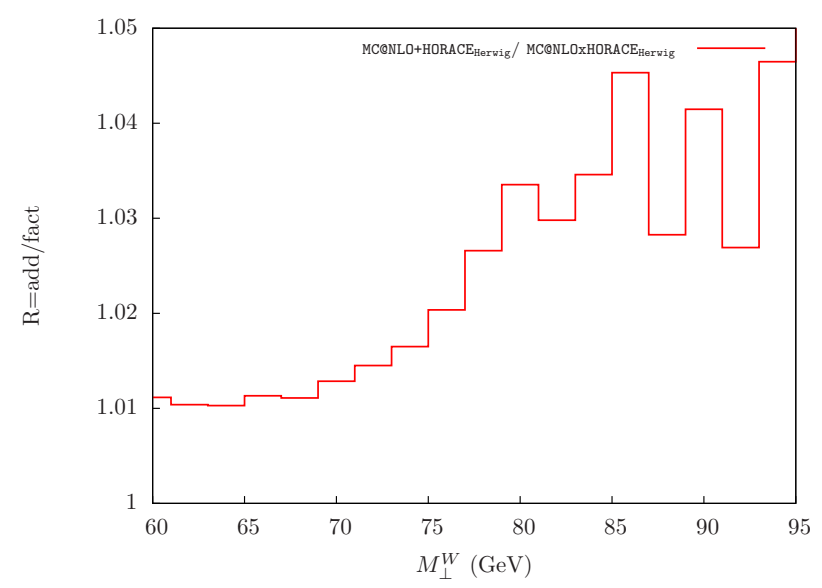

Figure 5: Ratio of the additive/factorized prescritpions for the $W$ transverse mass distribution.

In summary, the DY processes are used to extract a very precise determination of the $W$ boson mass and decay width. Since a precise extraction of these parameters relies on the accurate determination of the shape of the relevant distributions, it will be crucial to understand the impact of QCD higher orders and of the prescriptions for the combination of QCD and EW corrections.

\section{Acknowledgments}

$\mathrm{AV}$ wants to thank the organizers for the kind invitation to give this talk and for the very young and friendly atmosphere of the conference.

\section{References}

[1] G. Balossini et al., JHEP01(2010)013 [arXiv:0907.0276 [hep-ph]].

[2] "W mass workshop", Milano, March 18-19 2009, http://wwwteor.mi.infn.it/ vicini/wmass.html 
[3] F. Ambroglini et al., Proceedings of the Workshop on Monte Carlo's, Physics and Simulations at the LHC PART I, arXiv:0902.0293 [hep-ph];

F. Ambroglini et al., Proceedings of the Workshop on Monte Carlo's, Physics and Simulations at the LHC PART II, arXiv:0902.0180 [hep-ph] .

[4] C. Buttar et al., arXiv:0803.0678 [hep-ph].

[5] C. Balazs and C. P. Yuan, Phys. Rev. D56 (1997) 5558 [arXiv:hep-ph/9704258].

[6] F. Landry, R. Brock, P.M. Nadolsky and C.-P. Yuan, Phys.Rev.D67 (2003) 073016 [arXiv:hep-ph/0212159]

[7] G. Bozzi, S. Catani, G. Ferrera, D. de Florian and M. Grazzini, Nucl. Phys. B 815 (2009) 174 [arXiv:0812.2862 [hep-ph]].

[8] S. Catani, L. Cieri, G. Ferrera, D. de Florian, M. Grazzini, "Vector boson production at hadron colliders: a fully exclusive QCD calculation at NNLO", e-Print: arXiv:0903.2120 [hep-ph]

[9] S. Frixione and B. R. Webber, JHEP 0206 (2002)029 [arXiv:hep-ph/0204244].

[10] S. Alioli, P. Nason, C. Oleari and E. Re, JHEP 0807 (2008) 060 [arXiv:0805.4802 [hep-ph]].

[11] G. Corcella et al., "HERWIG 6.5 release note", [arXiv:hep-ph/0210213].

[12] T. Sjöstrand, P. Eden, C. Friberg, L. Lonnblad, G. Miu, S. Mrenna and E. Norrbin, Comput. Phys. Commun. 135 (2001) 238 [arXiv:hep-ph/0010017].

[13] C.M. Carloni Calame, G. Montagna, O. Nicrosini and A. Vicini, JHEP 0612 (2006)016, hep-ph/0609170, JHEP 0710 (2007) 109, [arXiv:0710.1722 [hep-ph]], and references therein.

[14] Q.-H. Cao and C.-P. Yuan, Phys.Rev.Lett.93(2004)042001.

[15] G. Balossini et al., Acta Phys. Polon. B 39 (2008) 1675 [arXiv:0805.1129 [hep-ph]].

[16] W. Hollik, T. Kasprzik and B. A. Kniehl, Nucl. Phys. B 790, 138 (2008) [arXiv:0707.2553 [hep-ph]].

[17] J. H. Kuhn, A. Kulesza, S. Pozzorini and M. Schulze, Nucl. Phys. B 797 (2008) 27 [arXiv:0708.0476 [hep-ph]];

J. H. Kuhn, A. Kulesza, S. Pozzorini and M. Schulze, Phys. Lett. B 651 (2007) 160 [arXiv:hep-ph/0703283].

[18] A. Denner, S. Dittmaier, T. Kasprzik and A. Muck, arXiv:0906.1656 [hep-ph]

[19] M.L. Mangano, M. Moretti, F. Piccinini, R. Pittau and A.D. Polosa, JHEP 0307 (2003) 001, hep-ph/0206293.

[20] John Campbell, Keith Ellis, Phys.Rev.D65:113007, hep-ph/0202176, Phys.Rev.D62:114012, hep-ph/0006304, Phys.Rev.D60:113006, hep-ph/9905386, http://mcfm.fnal.gov

[21] S. Catani and B. R. Webber, JHEP 9710 (1997) 005 [arXiv:hep-ph/9710333] and references therein. 